This document is the Accepted Manuscript version of a Published Work that appeared in final form in Journal of Physical Chemistry B, copyright (C) American Chemical Society after peer review and technical editing by the publisher.

To access the final edited and published work see: https://doi.org/10.1021/acs.jpcb.7b03475 


\title{
Addressing the Environment Electrostatic Effect on Ballistic Electron Transport in Large Systems: a QM/MM-NEGF Approach
}

\author{
Gustavo T. Feliciano, ${ }^{*} \dagger$ Carlos Sanz-Navarro, ${ }^{\ddagger}$ Mauricio Domingues \\ Coutinho-Neto, " Pablo Ordejón, ${ }^{\ddagger}$ Ralph H. Scheicher, ${ }^{\S}$ and Alexandre Reily \\ Rocha*,\| \\ $\dagger$ Instituto de Química, Departamento de Fisico-Química, Universidade Estadual Paulista \\ (UNESP), 14800-060, Araraquara, SP, Brazil \\ $\$$ Catalan Institute of Nanoscience and Nanotechnology (ICN2), CSIC and The Barcelona \\ Institute of Science and Technology, Campus UAB, Bellaterra 08193 Barcelona, Spain \\ ฯ Centro de Ciências Naturais e Humanas, Universidade Federal do ABC, 09210-580, \\ Santo André, São Paulo, Brazil \\ $\S$ Division of Materials Theory, Department of Physics and Astronomy, Uppsala University, \\ SE-751 20, Uppsala, Sweden \\ || Instituto de Física Teorica, Universidade Estadual Paulista (UNESP), 01140-070, São \\ Paulo, SP, Brazil \\ E-mail: gtroiano@iq.unesp.br; reilya@ift.unesp.br \\ Phone: +55 (16) 33019869
}

\section{Abstract}

The effects of the environment in nanoscopic materials can play a crucial role in device design. Particularly in biosensors, where the system is usually embedded in a solution, water and ions have to be taken into consideration in atomistic simulations of electronic transport for a realistic description of the system. In this work we present a methodology that combines quantum mechanics/molecular mechanics methods (QM/MM) with the non-equilibrium Green's function framework to simulate the electronic transport properties of nanoscopic devices in the presence of solvents. As a case in point we present further results for DNA translocation through a graphene nanopore. In particular we take a closer look into general assumptions in a previous work. For this sake, we consider larger QM regions that include the first two solvation shells and investigate the ef- fects of adding extra k-points to the NEGF calculations. The transverse conductance is then calculated in a prototype sequencing device in order to highlight the effects of the solvent.

\section{Introduction}

Manipulation of electronic properties of matter at the nanoscale could allow for the design of potentially revolutionary devices. In the particular case of biosensors - where a biological molecule interacts with an analyte for detection - the dimensions of the device are compatible with biological molecules, and one can envision sensing reaching the single-molecule level. A particular type of biosensor is a DNA sequencing device. ${ }^{1,2}$ This is a process where the order of the nucleobases in a DNA molecule is determined, depending on the interaction of each nucleotide with a given sensor device. Recently, 
nanopores have been heralded as the path towards single-molecule sequencing. ${ }^{3-9}$ In such devices, the DNA molecule is driven through a pore, where each nucleobase can selectively interact with the device. Detection is then obtained by measuring the current, while a particular nucleotide is present in the pore, i.e. using a resistive biosensor. In principle, one can use either biological entities for detection, such as protein translocation channels or non-biological ones, such as silicon nitride or graphene nanopores. ${ }^{2}$

Typically, biological molecules are immersed in a solution containing a solvent - mostly water and salt, in different concentrations. Removal from this solution, in many cases, leads to significant changes in structure and function. ${ }^{10}$ Thus, in the design of a biosensor, the dielectric effect of the solvent might be crucial. ${ }^{11}$ Fluctuations in the atomic configuration of the environment most likely change the potential seen by the device, which in turn could alter the behavior of the current-voltage curve in a resistive biosensor.

From the theoretical point of view, performing $a b$ initio molecular dynamics (AIMD) of the full system whilst simulating the electronic transport is, up to the present point, still prohibitively expensive to be carried out. A possible workable strategy is to separate the structure generation step from the transport calculation step. Empirical molecular dynamics simulation (MD) is employed to sample over possible atomic configurations that represent the system, and then Density Functional Theory (DFT) is used ${ }^{12,13}$ to obtain the electronic structure and the system Hamiltonian. Transport calculations are then performed within the non-equilibrium Green's Function (NEGF) method. ${ }^{14}$

Still, with a few exceptions ${ }^{15}$, the solvent is only taken into account in the configurational sampling. Thus, the environment is usually not explicitly considered in the electronic structure / electron transport calculations, especially when the calculation involves biological systems. ${ }^{16-18}$ In one of the first works to try to address the effect of a dielectric environment on electron transport considering up to 360 wa- ter molecules explicitly in the electron transport calculation. ${ }^{19}$ There it was demonstrated that, when the molecular system attached to the electrodes is polar, the environment screens part of the electric dipole, causing shifts in the transmission spectrum. When the molecule is non-polar, the time-averaged electric field from the environment is close to zero, and the average shift in the transmission is much smaller. At the same time, different transport regimes are investigated, by means of model Hamiltonians combined with classical molecular dynamics simulations, in order to reduce the computational cost and improve the phase space sampling. ${ }^{20-24}$.

Given that the number of atoms present in biosensor models is very large once the environment is explicitly taken into account, additional approximations must be employed. The hybrid QM/MM approach ${ }^{25-27}$ divides the system Hamiltonian into a QM region, where atoms are treated quantum mechanically, and a MM region, where atoms are treated by classical force field methods. This partition allows for the treatment of very large systems. The interaction between the two sub-systems is included by using typical classical Coulomb and van der Waals terms so that the environment is represented by an external electrostatic potential for the QM part.

In a recent work ${ }^{28}$, a NEGF methodology allied to a QMMM description of the environment was used, in order to elucidate the effect of many water molecules, counterions and a few nucleobases on the zero-bias conductance of a graphene-based DNA sequencing device. The chosen system is one of the most promising systems for DNA sequencing and detection, and yet, due to its small thickness, it is also highly exposed to the environment. In this particular study, the chosen QM system was only the nucleotide and the nanopore. It was shown that the solvent modulates the charge transfer between the nucleobase and the nanopore, and the precise environment description is crucial to understand the working mechanism of the sensor.

The effect of the environment can be seen as an electrostatic gating effect, since DNA is 
negatively charged in aqueous solution at neutral $\mathrm{pH}$. This charge induces reorganization of the solvent and counterions around the charge, which changes the energy and the alignment of the electronic states of the molecule. In gasphase calculations this is usually not taken into consideration, and can only be fully addressed self-consistently when DNA is interacting with water and the nanopore. These specific changes cause shifts in the transmission function. The same conductance shift has also been observed by Runger et al. in carbon nanotubes in water $^{19}$.

In this work, we propose a deeper analysis in the NEGF-QMMM electron transport calculation protocol, with a detailed analysis on the effect of the environment on specific details of the electronic structure and electrostatic potential, and also including k-point sampling in the transport calculation, and also the effect of other alternative QM/MM partitions, including water molecules in the QM region, offering more insight in the QM/MM-NEGF protocol.

The protocol was applied to the study of the electron transport across a graphene sheet containing a nanopore through which a DNA molecule is translocated, as a test case. This system has been heralded as a potential setup for electronic DNA sequencing. The general idea is that a graphene sheet containing a nanopore can be used as a sieve for DNA. Concomitantly the conductance on graphene can be measured and the nucleobase can be differentiated. This is a situation where the effects of the chemical environment are potentially significant, and one ideally suited for this methodology.

\section{Computational details}

The prototypical device we are considering consists of two metallic terminals coupled via a socalled scattering region. In the particular case of a nanopore used for DNA sequencing, the electrodes consist of pristine graphene (a unit cell of the semi-infinite electrodes and the scattering region would, in principle, be a graphene sheet containing the nanopore, a strand of DNA that is sieved through the pore, water molecules and the counter-ions. A typical setup is shown in Figure 1.

In order to obtain the electronic transport properties one can use a Green's function approach to obtain the low-bias conductance $^{14,29-31}$

$$
\sigma=\frac{2 e^{2}}{h} T\left(E_{F}\right)
$$

where $G_{0}=2 e^{2} / h$ is the quantum of conductance, and $T\left(E_{F}\right)$ is the total transmission at the Fermi level. In order to do this, the open system is partitioned in three: a central scattering region and two electrodes. Typically, the electrodes are semi-infinite periodic structures. Figure 1 illustrates the system under study, highlighting the position of the electrodes.

In turn, the transmission can be obtained via Green's functions for the open system ${ }^{32-35}$

$$
T(E)=\Gamma_{L}(E) G^{\dagger}(E) \Gamma_{R}(E) G(E),
$$

where $\Gamma_{\alpha}=i\left[\Sigma_{\alpha}-\Sigma_{\alpha}^{\dagger}\right](\alpha \equiv\{\mathrm{L}, R\}), \Sigma_{L / R}$ are the self-energies ${ }^{14,36,37}$ - the effect of the semiinfinite electrodes on the scattering region - and

$$
G^{R}(E)=\left[\epsilon^{+} S_{S}-H_{S}-\Sigma_{L}(E)-\Sigma_{R}(E)\right]^{-1},
$$

is the retarded Green's function for the scattering region, and $\epsilon^{+}=E+i \eta$. The key point is to obtain the QM Hamiltonian $H_{S}$ (and overlap matrix $S_{S}$ ) describing the scattering region. Ideally this would entail writing a single particle Hamiltonian in a localized basis set for all the atoms shown in Figure 1.

Furthermore, given the dynamic nature of the problem, one would require sampling over a set of structural configurations. For this sake, a classical parameterization of the system's Hamiltonian is employed for the time evolution of the atomic coordinates. The atomic configuration of the system is obtained from a classical molecular dynamics trajectory, which is a technique accurate enough to significantly sample the configurational space.

Given the atomic coordinates of the system, its electronic structure can be obtained. Further simplification of the problem is possible if 


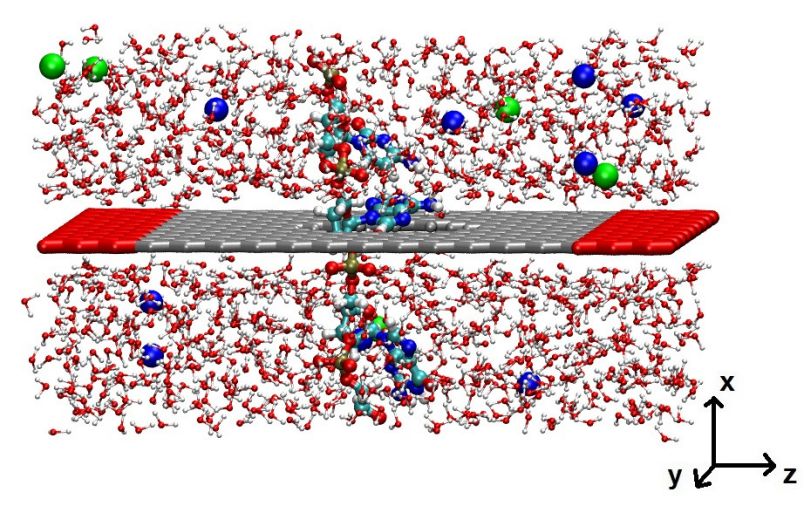

Figure 1: Atomistic Illustration of the system in which the QM/MM-NEGF protocol was applied, composed by graphene, DNA, water and counterions. The electrode region, at the extremities of the graphene sheet, is highlighted in red. Sodium atoms are in blue and chloride atoms are in green

we note that electron flow occurs in a limited region, in the case of a device in aqueous solution. The effect of most of the solvent is electrostatic in nature. The same problem is faced in a number of problems involving biomolecules. This is dealt with by using a hybrid quantum mechanics/molecular mechanics partition, ${ }^{26}$ that is, an environment described by classical force field partial charges acting electrostatically over a subset of the system described by quantum mechanics, where electrons are explicitly taken into account. This is especially valid, as long as electronic reorganization is restricted to the quantum region (QM). In this sense, the classical region (MM) creates a potential that only polarizes the quantum charge density, and no charge is transferred between the regions. ${ }^{38,39}$

The quantum-mechanical subsystem is usually treated by first-principles calculations based on density functional theory (DFT). ${ }^{12,13}$, and the MM electrostatic potential is evaluated from the force field's partial charges, using Coulomb's law. In our case, the MM electrostatic potential was calculated using a QM/MM implementation in SIESTA, ${ }^{40,41}$. The MM potential is also fully periodic, with the same lattice vectors of the QM system. In the DFT framework, the calculated potential is directly added to the Hartree potential, until self-consistency is achieved in the electronic charge density of the QM region. The resulting Kohn-Sham Hamiltonian can then be used in equation 3 for the electron transport calculation.

When the QM/MM boundary is defined across a covalent bond, a specific description for the frontier must be used. The most common solution is the scaled position link-atom method $(\mathrm{SPLAM})^{42}$, where the valence of the QM region is completed by adding hydrogen atoms along the frontier bond and the MM frontier charge is corrected, in order to avoid doublecounting effects on the potential.

Finally, it is important to note that the electron transport calculation requires well defined boundary conditions between the scattering region and the electrodes. Since the external potential in this region can fluctuate due to the presence of the solvent, we smoothly truncated the MM potential to zero at this region, using a Fermi-Dirac-like smoothing function. This guarantees a smooth matching between the scattering region and the left and right electrodes, which are taken as solvent-free regions.

In essence, we perform a three-step procedure, namely first a classical molecular dynamics simulation to obtain a set of configurations. They are subsequently used, by appropriately partitioning the system, in a single point QM/MM calculation. With the resulting Kohn-Sham Hamiltonian we calculate the transmission probability as a function of energy.

\section{Results}

\section{System construction and configu- rational sampling}

The graphene nanopore is constructed from a square-shaped graphene sheet of dimensions 4 $\mathrm{nm} \times 4 \mathrm{~nm}$ containing a nanopore, with a central hexagonal-shaped orifice, approximately $1.3 \mathrm{~nm}$ wide, with the edges in the zigzag configuration. The size of the nanopore was chosen as a compromise between experimental fabrication 
feasibility ${ }^{43,44}$ and consideration for the computational expenses of the simulations. The dangling bonds on the edge were saturated with hydrogen atoms. The graphene nanopore is composed of 615 atoms (see Figure 1).

A periodic single-stranded DNA molecule with 4 nucleotides (ATCG sequence) was inserted in the nanopore, and the system was completely immersed in water (4400 atoms) with $8 \mathrm{Na}^{+}$and $4 \mathrm{Cl}^{-}$counter ions at $0.1 \mathrm{M}$ concentration. The imbalance of 4 extra $\mathrm{Na}^{+}$ ions compensates the 4 negative charges from the DNA phosphate groups. (Figure 1).

As previously described, the configurational space is sampled by classical molecular dynamics (MD) simulations, employing the standard all-atom version of the AMBER99SB ${ }^{45}$ empirical force field, implemented in the GROMACS ${ }^{46}$ package, and the Particle-Mesh-Ewald $(\mathrm{PME})^{47}$ method is employed for the calculation of the electrostatic energy. We used the SPC water model ${ }^{48}$ and parameters for benzene to model graphene, with partial charges only in the hydrogen atoms terminating the nanopore edges and their neighboring carbon atoms.

Four initial configurations are considered for DNA: in each, one of the four nucleotides is close to the pore. MD simulations are performed for each initial configuration, yielding 4 MD simulations. The system undergoes a thermalization procedure performed for 100 ps at $300 \mathrm{~K}$ using an NVT ensemble. We then equilibrated the water density for a further 200 ps using an NPT ensemble at $300 \mathrm{~K}$ and 1 bar with a Nose-Hoover thermostat ${ }^{49,50}$ and ParrinelloRahman barostat ${ }^{51}$. In both cases, each nucleotide is restricted inside the pore by a harmonic potential restriction applied at the $\mathrm{x}$ coordinate of the DNA molecule backbone atoms, using a spring constant of $1000 \mathrm{KJ} \mathrm{mol}^{-1} \mathrm{~nm}^{-2}$, allowing for the nucleobase nucleobase in the pore to move freely in the plane yz. Finally, in the production stage, a $300 \mathrm{~K}$ NVT 2000 ps MD simulation is performed in which all the atoms are free to move.

From the MD simulations, one frame is selected for each nucleotide. This frame is selected in such a way that the transmission lies close to the average transmission of 200 curves, for each nucleotide, from the previous work ${ }^{28}$. This structure is taken as input for the following calculations. This choice was employed for the sake of obtaining a clear picture of the differences in the electronic structure and transmission when changing the QM/MM partition and including explicit water molecules in the QM region, and at this stage, extensive sampling of QM - QM/MM electronic structure calculations is not required.

\section{Electronic structure, QM/MM partition and NEGF calculations}

Subsequently, the electronic structure of the system is obtained for each frame from the MD simulations. Two different QM/MM partitions are considered in this work, regarding the QM region composition, the partitions are: (A) graphene nanopore and one nucleotide, illustrated in Figure 2a (B) graphene nanopore, one nucleotide and a layer of water molecules within $8 \AA$ of the nucleotide, comprising 108 water molecules, illustrated in Figure 2b. In each partition, the MM region contains the remainder of the system (water, counterions and remaining nucleotides).

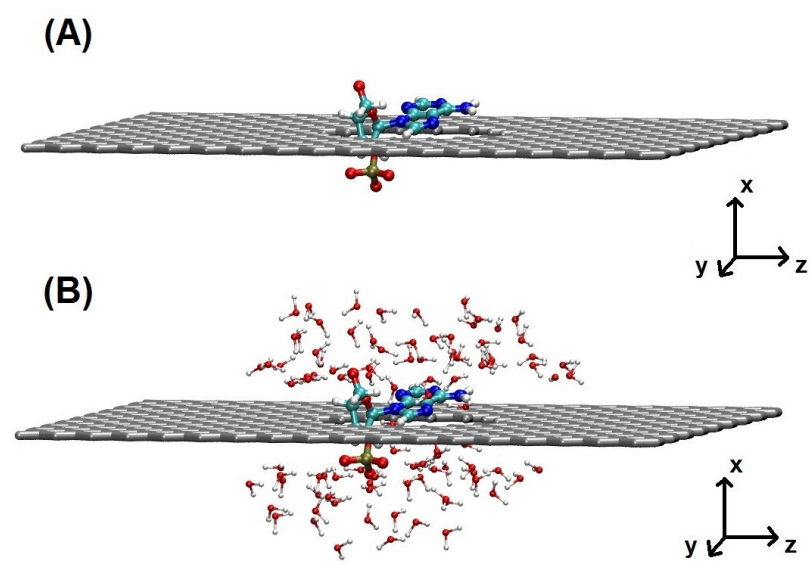

Figure 2: Different QM/MM partitions explored in this study: (A) QM/MM partition A, composed by graphene and a nucleobase (B) QM/MM partition B, composed by graphene, a nucleobase and a $8 \AA$ layer of surrounding water molecules. 
The QM/MM covalent boundary is established across two chemical bonds in DNA, in the phosphate group connecting the QM nucleotide with its two neighbors, as shown in figure 3. The link atoms are placed between the oxygen of one nucleotide and the carbon atom of the sugar ring linking to the next nucleotide. Thus, the QM system always has an overall 1e charge, and the MM environment has a +1 e charge.

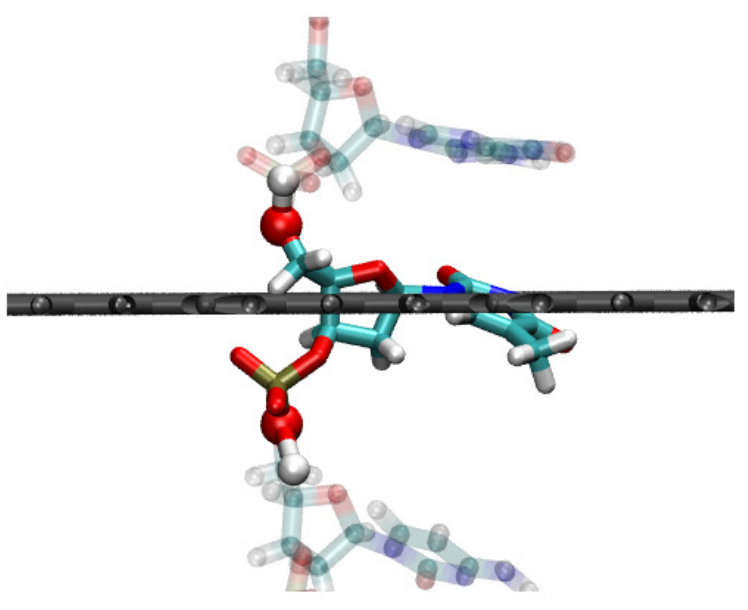

Figure 3: The frontier QM/MM covalent bonds defined for this work. The transparent part represents the DNA region described by the classical Hamiltonian. The link atoms are emphasized as spheres in the opaque region

The quantum region (QM) is described using the generalized gradient approximation for the exchange and correlation potential in its PBE form ${ }^{52}$. Core electrons were replaced by norm-conserving pseudopotentials, fully factorized and using non-linear partial-core corrections for pseudo-wave-function smoothness close to the nuclei. The Kohn-Sham wavefunctions for the valence electrons were expanded in a basis set of soft-confined, finitesupport numerical atomic orbitals. A double$\zeta$ polarized (DZP) basis set was used for the nucleotide atoms and a double- $\zeta$ (DZ) basis for the graphene membrane. The calculations were carried out with the SIESTA code ${ }^{40}$, using a finite real-space grid corresponding to a 200 Ry plane-wave cutoff for the integrals in real space. The electrodes $(\mathrm{L} / \mathrm{R})$ are taken as pristine graphene sheets whereas the scattering region $(\mathrm{S})$ consists of a piece of graphene containing the pore and one nucleobase.

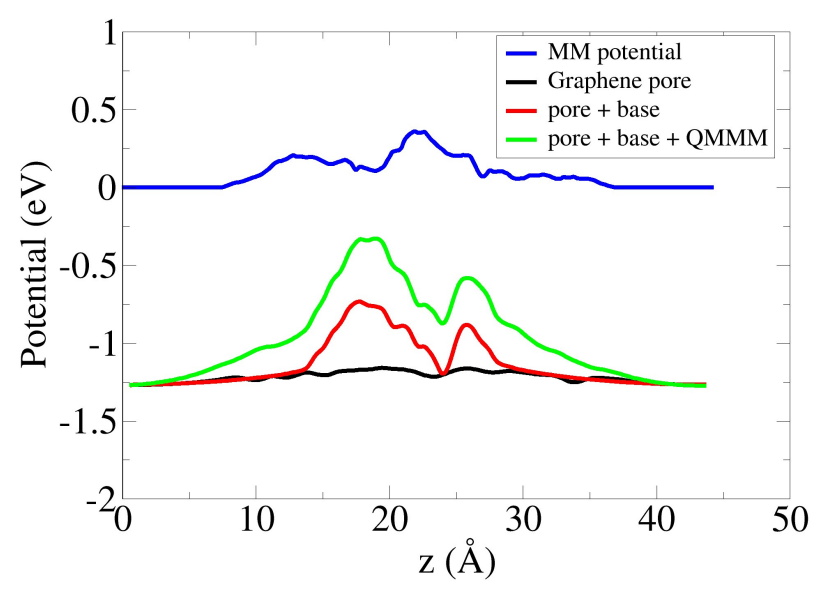

Figure 4: Macroscopically averaged electrostatic potential as a function of the $\mathrm{z}$ coordinate of one of the atomic configurations representing the graphene-nucleobase system, comparing the effect of each part of the system (bare pore, pore + nucleobase and pore + nucleobase + MM potential)

The classical region (MM) is described by the AMBER99SB force field parametrization. QM/MM interaction cutoff is $25.0 \AA$ which is large enough to take all the remaining atoms in the MD frame into account in the classical region. The MM Coulomb potential is calculated by an Ewald summation.

Further analysis of the MM electrostatic potential, as shown in figures 4 and 5 reveals the extent of the environment action on the graphene/nucleobase system, along with the charge density difference. Figure 4 shows the macroscopically averaged electrostatic potential. Since the QM system is negatively charged, the MM potential is predominantly positive. As depicted by the colored surface maps, the electronic charge follows the external potential pattern, extending all over the graphene nanopore and the nucleobase. The potentials, however, are not additive, since the green curve comes from the electronic structure calculation under the MM potential, and therefore, the electronic structure is not the same as 

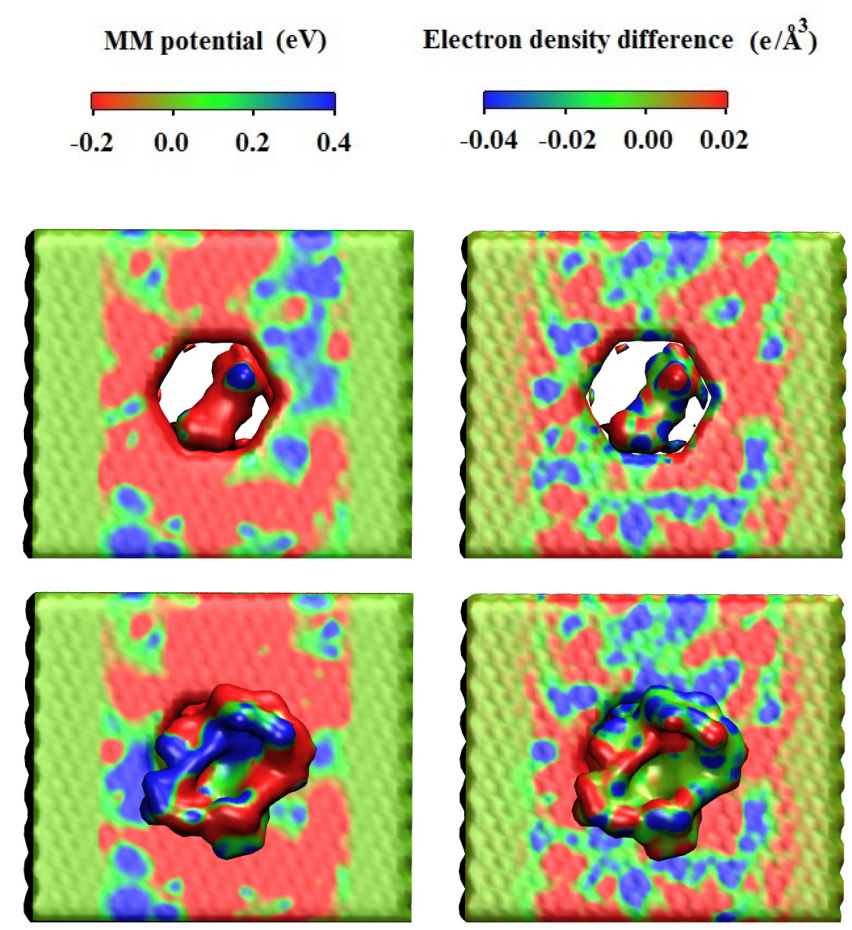

Figure 5: Surface map of the electrostatic potential generated by the MM classical charges in QM/MM partition A (top left) and B (bottom left) for a particular nucleotide. Charge density difference upon inclusion of the QM/MM potential (top right and bottom right). Both maps are projected on an electronic isodensity surface of $10^{-4} \mathrm{e} / \AA^{3}$

the calculation without the MM potential.

Figure 6 depicts the electronic projected density of states of the QM system in the chosen snapshots, under the QM/MM partition A. Without the QM/MM inclusion, there are electronic states of the phosphate group, close to the Fermi level. Upon inclusion of the MM potential, these states change in energy, as the phosphate negative charge is stabilized on the oxygen atoms. Some states generated from hybridization of the orbitals on the nucleobase region get closer to the Fermi level from positive values, which have a significant contribution from nitrogen and carbon atoms from the nucleobase ring moieties.

Figure 7 illustrates the projected density of states, but in the QM/MM partition B. Now the system has water molecules in the QM region, and display a remarkable contribution very close to the Fermi level, in the absence of the MM potential. In the presence of the MM potential, the water states also redistribute in energy. Comparing to the results of the partition A, the inclusion of water molecules in the QM region already change the behavior of the phosphate moiety, and the negative charge is stabilized even without the MM potential. However, many other states from the QM water molecules appear close to the Fermi level, which can be associated to DFT's self interaction problem, already reported in similar studies ${ }^{19}$. Inclusion of the MM potential on partition B moves these states away from the Fermi level, and describe the same shift in the nucleobase electronic states as the QM/MM calculation on partition A. What can be concluded is that the energetic alignment of the electronic states that have contribution from the nucleobase is similar in both QM/MM partitions, and the overall density of states remains the same. Inclusion of the water molecules in the QM region, allied to a reasonable description of the environment (with QMMM) shows that water molecules have a significant contribution to the density of states far from the Fermi level, and this should be important only when investigating the transmission properties of the device out of the zero-bias limit. This observation agrees with the previous work, showing that the QM/MM description is enough to capture the most important effects of the environment in the zero-bias limit, and at the same time, if desirable, describe the influence of the electronic states of water, with the correct alignment.

Figure 8 shows the transmission spectrum results, for each of the selected snapshots (one for each nucleobase), under each QM/MM partition, always comparing with the transmission spectrum of the corresponding isolated nanopore. At the Fermi level, there are differences of the order of 0.02 , but the differences are significantly larger when looking at the spectrum at other energies. This means that either external gating or higher applied source-drain bias could give rise to significant changes.

In can be seen that the presence of the nucleotide, along with the MM potential leads to changes in the charge distribution around the pore, and consequently to changes in the 

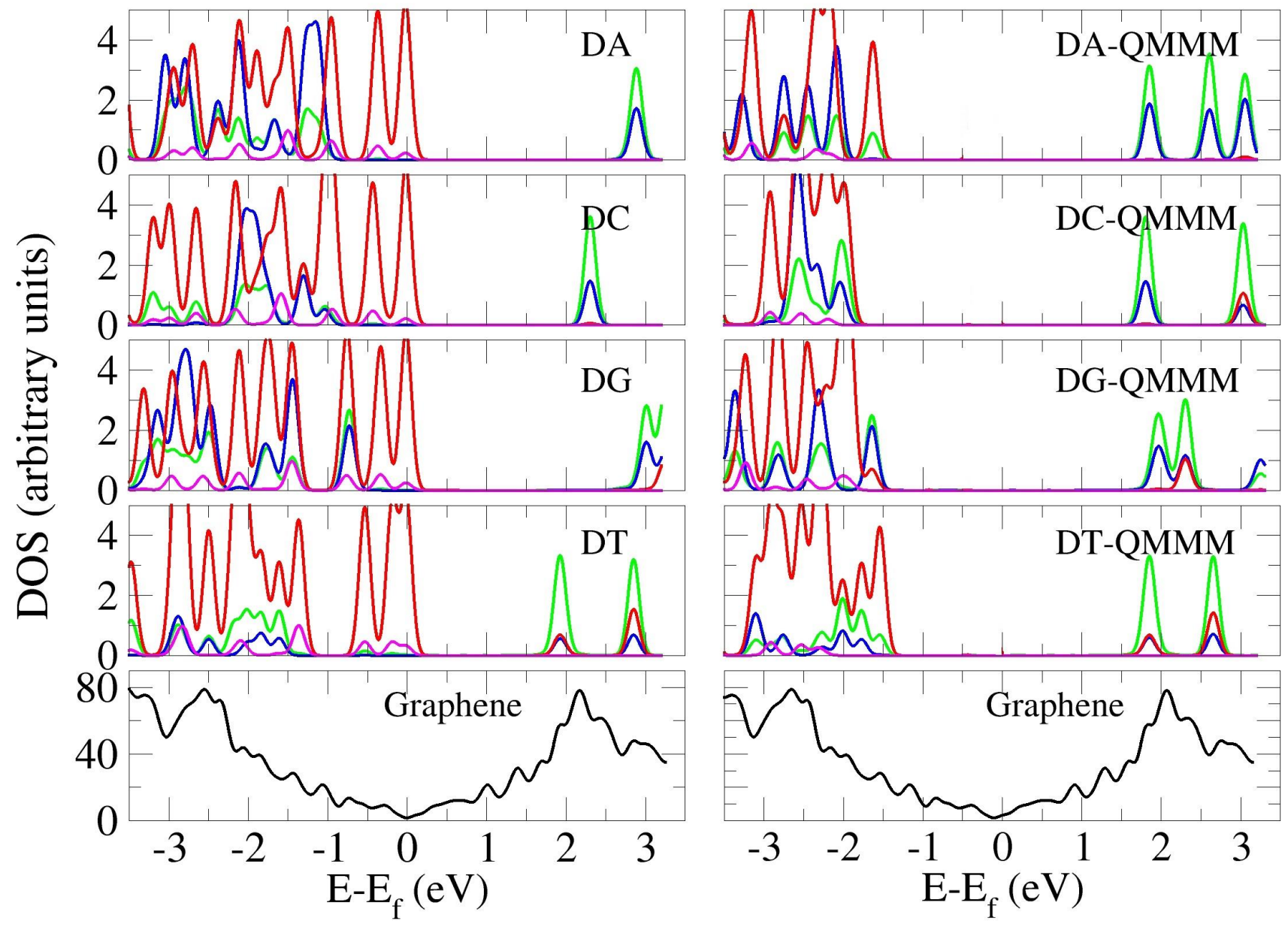

Figure 6: Projected DOS of the graphene-nucleobase systems, for the QM/MM partition A

conductance, since the PDOS analysis shows negligible contribution of the nucleobase states at the electrode Fermi level in all studied QM/MM partitions. Zero-bias conductance changes come mainly from the shift of the graphene states lying close to the electrode Fermi level, in response to the changes in the electronic structure of the DNA and the graphene pore, which in turn come from the inclusion of the MM potential.

Therefore all the effects seen in the calculation suggest an electrostatic gating role for the environment. The change in the charge of the DNA and the atoms in the nanopore in turn alter the position of the electronic states involving graphene. Such feature is easily taken into account under the proposed methodology.

\section{Conclusions}

In this work, we employ the QM/MM-NEGF electron transport calculation protocol for a detailed decription of the effect of the environment over the transmission properties of a graphene-based DNA electronic sequencing device. There are significant effects that the model can capture upon the inclusion of the environment, and the method allows for the inclusion of large systems (composed by thousands of atoms) with low computational cost. Another interesting advantage is that the parametrized MM charges, based on high level quantum chemical calculations and potential fitting to classical charges, enforce the expected distribution of charge, avoiding DFT excessive charge delocalization, where the MM potential, being non-polarizable, acts as a constraint for the QM 

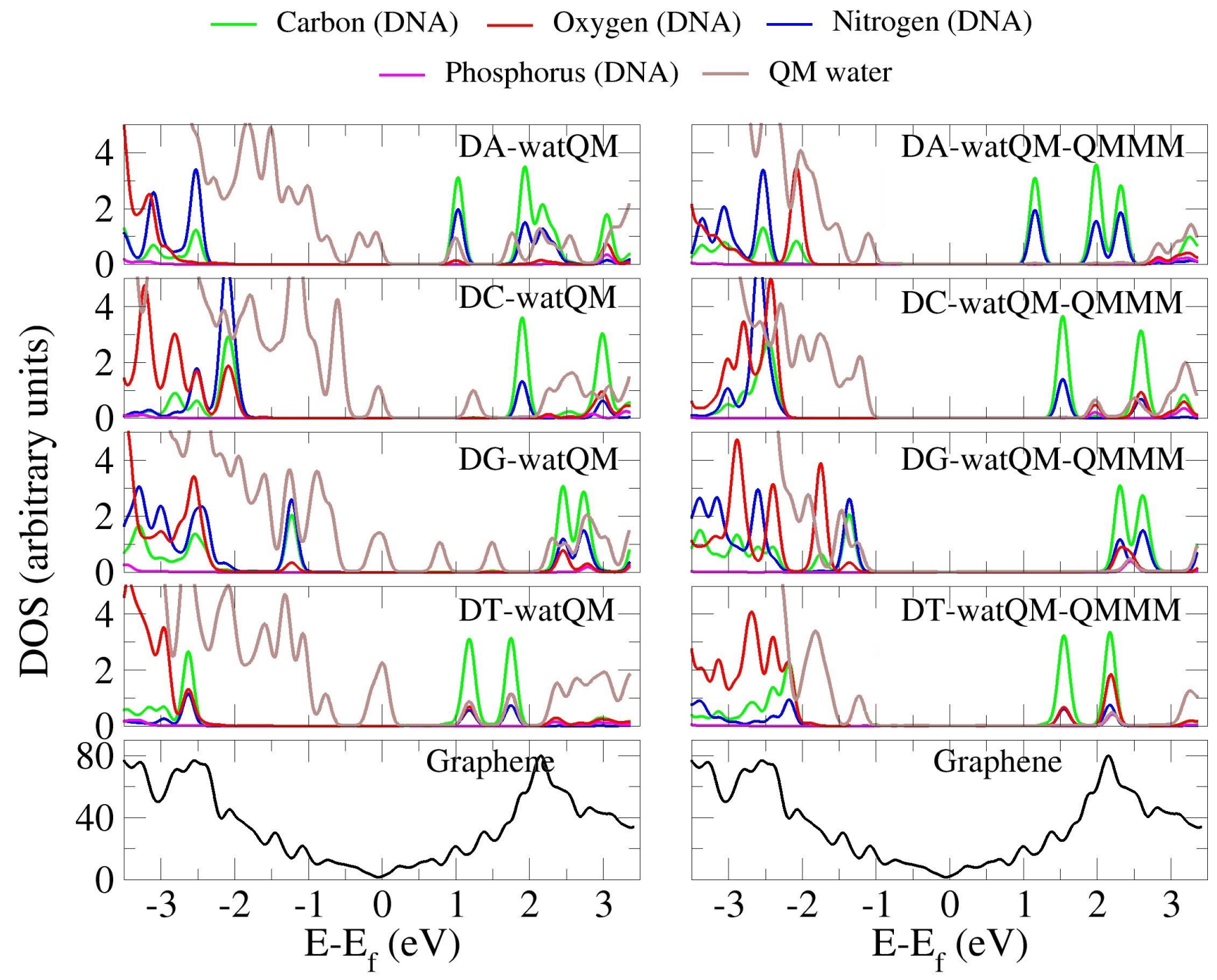

Figure 7: Projected DOS of the graphene-nucleobase systems, for the QM/MM partition B. The DOS from water molecules in the QM region are downscaled by a factor of 5

electron density. We also showed that the technique can be adapted to study the explicit contribution of the solvent/environment orbitals to generate the transmission channels, just by extending the QM/MM partition. However, the extent of the QM region is, by definition, not unique, and should be systematically tested.

Another possible interesting improvement over the current implementation is the use of a polarizable force field in the molecular dynamics simulation, for a description of the point charges or the MM region, when the environment is significantly polarizable. Force field parameterization based on symmetry adapted perturbation theory is an interesting way to obtain accurate structures for the methodology, as the polarization can be taken into account in all phases of the simulation, at low computational effort. ${ }^{53}$ All improvements can be incorporated in the current methodology to provide a reasonable description of the electrostatic effect of the environment on electron transport properties, as proposed in the QM/MM-NEGF protocol.

\section{Acknowledgments}

The authors thank FAPESP and UFABC for financial support. A. R. R. acknowledges support from ICTP-SAIFR (FAPESP project 2011/11973-4) and the ICTP-Simons Foundation Associate Scheme. Computer time was provided by IFT/Unesp and USP/Sampa institutions. R.H.S. thanks the Swedish Research 

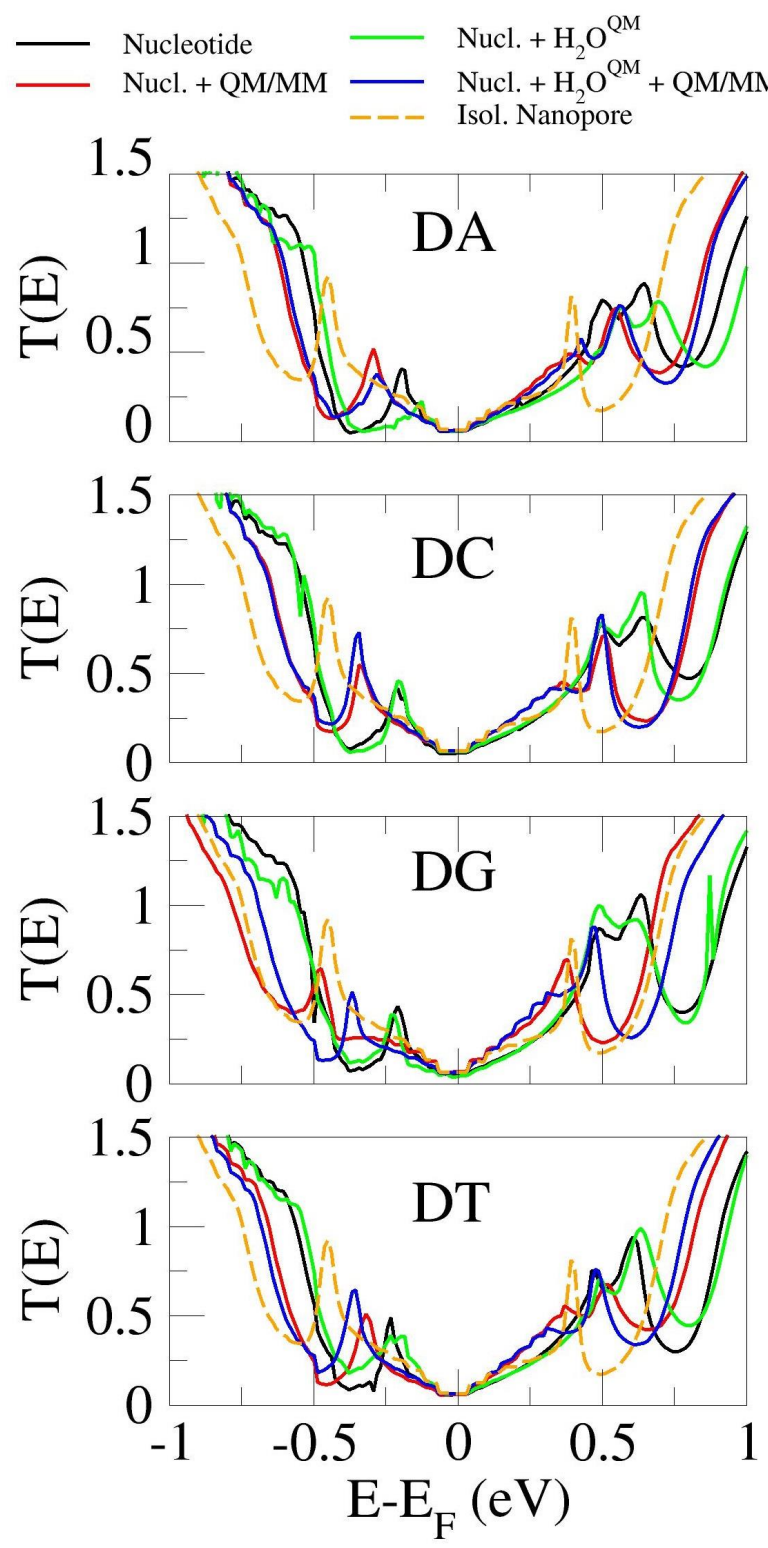

Figure 8: Zero-bias transmission for all studied nucleobases, with 10 k-points, in the configurations: pure graphene (black) and graphene+nucleobase system in 4 situations: bare system (red), system + MM potential (blue), system + quantum water layer (green), system with quantum water layer with MM potential (orange)

Council for financial support. PO acknowledges support from the Spanish MINECO (Grant FIS2015-64886-C5-3-P and the Severo Ochoa Centers of Excellence Program Grant SEV-2013-0295), Generalitat de Catalunya Government (2014SGR301), and EU H2020EINFRA-5-2015 MaX Center of Excellence (Grant 765986). C.S.N. acknowledges support from MINECO through the Ramon y Cajal Program.

\section{References}

(1) Vercoutere, W.; Akeson, M. Biosensors for \{DNA\} sequence detection. Curr. Opin. Chem. Biol. 2002, 6, $816-822$.

(2) Heerema, S. J.; Dekker, C. Graphene nanodevices for DNA sequencing. Nat. Nanotechnol. 2016, 11, 127-136.

(3) Clarke, J.; Hai-Chen,; Jayasinghe, L.; Patel, A.; Reid, S.; Bayley, H. Continuous base identification for singlemolecule nanopore DNA sequencing. Nat. Nanotechnol. 2009, 4, 265-270.

(4) Postma, H. W. C. Rapid Sequencing of Individual DNA Molecules in Graphene Nanogaps. Nano Lett. 2010, 10, 420-425, PMID: 20044842.

(5) Feng, J.; Liu, K.; Bulushev, R. D.; Khlybov, S.; Dumcenco, D.; Kis, A.; Radenovic, A. Identification of single nucleotides in MoS2 nanopores. Nat. Nanotechnol. 2015, 10, 1070-1076.

(6) Niedzwiecki, D. J.; Lanci, C. J.; Shemer, G.; Cheng, P. S.; Saven, J. G.; Drndic, M. Observing Changes in the Structure and Oligomerization State of a Helical Protein Dimer Using Solid-State Nanopores. ACS Nano 2015, 9, 89078915.

(7) Di Ventra, M.; Taniguchi, M. Decoding DNA, RNA and peptides with quantum tunnelling. Nat. Nanotechnol. 2016, 11, 117-126.

(8) Deamer, D.; Akeson, M.; Branton, D. Three decades of nanopore sequencing. Nat. Biotechnol. 34, 518-524.

(9) de Souza, F. A. L.; Amorim, R. G.; Scopel, W. L.; Scheicher, R. H. Electrical detection of nucleotides via nanopores in a hybrid graphene/h-BN sheet. Nanoscale 2017, 9, 2207-2212. 
(10) P. W. Fenimore, B. H. M. F. G. P., H. Frauenfelder Slaving: Solvent Fluctuations Dominate Protein Dynamics and Functions. Proc. Natl. Acad. Sci. U. S. A. 2002, 99, 16047-16051.

(11) Aryal, B. P.; ; Benson*, D. E. Electron Donor Solvent Effects Provide Biosensing with Quantum Dots. J. Am. Chem. Soc. 2006, 128, 15986-15987, PMID: $\overline{17165722 .}$

(12) Hohenberg, P.; Kohn, W. Inhomogeneous electron gas. Phys. Rev. 1964, 155.

(13) Kohn, W.; Sham, L. J. Self-Consistent Equations Including Exchange and Correlation Effects. Phys. Rev. 1965, 140, A1133.

(14) Datta, S. Electronic Transport in Mesoscopic Systems, 1st ed.; Cambridge University Press: Cambridge, 1995; pp $57-88$.

(15) Krems, M.; Zwolak, M.; Pershin, Y. V.; Di Ventra, M. Effect of Noise on DNA Sequencing via Transverse Electronic Transport. Biophys. J. 2009, 97, 1990-1996.

(16) Lagerqvist, J.; Zwolak, M.; Di Ventra, M. Fast DNA sequencing via transverse electronic transport. Nano Lett. 2006, 6, 77982.

(17) McFarland, H. L.; Ahmed, T.; Zhu, J.X.; Balatsky, A. V.; Haraldsen, J. T. First-Principles Investigation of Nanopore Sequencing Using Variable Voltage Bias on Graphene-Based Nanoribbons. J. Phys. Chem. Lett. 2015, 6, 2616-2621, PMID: 26266743.

(18) Prasongkit, J.; Feliciano, G.; Rocha, A. R.; He, Y.; Osotchan, T.; Ahuja, R.; Scheicher, R. H. Theoretical assessment of feasibility to sequence DNA through interlayer electronic tunneling transport at aligned nanopores in bilayer graphene. Sci. Rep. 2015, 5, 17560.
(19) Rungger, I.; Chen, X.; Schwingenschlögl, U.; Sanvito, S. Finite-bias electronic transport of molecules in a water solution. Phys. Rev. B 2010, 81, 235407.

(20) Gutierrez, R.; Caetano, R. A.; Woiczikowski, B. P.; Kubar, T.; Elstner, M.; Cuniberti, G. Charge Transport through Biomolecular Wires in a Solvent: Bridging Molecular Dynamics and Model Hamiltonian Approaches. Phys. Rev. Lett. 2009, 102, 208102.

(21) Gutierrez, R.; Caetano, R.; Woiczikowski, P. B.; Kubar, T.; Elstner, M.; Cuniberti, G. Structural fluctuations and quantum transport through DNA molecular wires: a combined molecular dynamics and model Hamiltonian approach. New J. Phys. 2010, 12, 023022.

(22) Woiczikowski, P. B.; Kubar, T.; Gutierrez, R.; Caetano, R. A.; Cuniberti, G.; Elstner, M. Combined density functional theory and Landauer approach for hole transfer in DNA along classical molecular dynamics trajectories. J. Chem. Phys. 2009, 130, 215104.

(23) Woiczikowski, P. B.; Kubar, T.; Gutierrez, R.; Cuniberti, G.; Elstner, M. Structural stability versus conformational sampling in biomolecular systems: Why is the charge transfer efficiency in G4-DNA better than in double-stranded DNA? J. Chem. Phys. 2010, 133, 035103.

(24) Popescu, B.; Woiczikowski, P. B.; Elstner, M.; Kleinekathofer, U. TimeDependent View of Sequential Transport through Molecules with Rapidly Fluctuating Bridges. Phys. Rev. Lett. 2012, 109, 176802.

(25) Sanz-Navarro, C. F.; Grima, R.; García, A.; Bea, E. a.; Soba, A.; Cela, J. M.; Ordejón, P. An efficient implementation of a QM-MM method in SIESTA. Theor. Chem. Acc. 2010, 128, 825-833. 
(26) Warshel, A.; Levitt, M. Theoretical studies of enzymic reactions: Dielectric, electrostatic and steric stabilization of the carbonium ion in the reaction of lysozyme. J. Mol. Biol. 1976, 103, 227-249.

(27) Sabin, J. R.; Brandas, E. Combining Quantum Mechanics and Molecular Mechanics. Some Recent Progresses in QM/MM Methods. Adv. Quantum Chem. 2010, 59, 1-416.

(28) Feliciano, G. T.; Sanz-Navarro, C.; Coutinho-Neto, M. D.; Ordejón, P.; Scheicher, R. H.; Rocha, A. R. Capacitive DNA Detection Driven by Electronic Charge Fluctuations in a Graphene Nanopore. Phys. Rev. Appl. 2015, 3, 034003.

(29) Haug, H.; Jauho, A. P. Quantum Kinetics in Transport and Optics of $\overline{\text { Semiconductors; Springer Series in Solid- }}$ State Sciences; Springer Verlag: New York, 1999; pp 1-362.

(30) Rocha, A. Theoretical and Computational Aspects of Electronic Transport at the Nanoscale; Trinity College, 2007.

(31) Brandbyge, M.; Mozos, J.-L.; Ordejón, P.; Taylor, J.; Stokbro, K. Density-functional method for nonequilibrium electron transport. Phys. Rev. B 2002, 65, 165401.

(32) Landauer, R. Spatial Variation of Currents and Fields Due to Localized Scatterers in Metallic Conduction 1, 233 (1957). IBM J. Res. Develop. 1957, 1, 233.

(33) Büttiker, M.; Imry, Y.; Landauer, R.; Pinhas, S. Generalized many-channel conductance formula with application to small rings. Phys. Rev. B 1985, 31, 6207.

(34) Landauer, R. Conductance from Transmission: Common Sense Points. Phys. Scr. 1992, 110, 1402.

(35) Caroli, C.; Combescot, R.; Nozieres, P.; Saint-James, D. Direct calculation of the tunneling current. J. Phys. C: Solid State Phys. 1971, 4, 916.
(36) Rocha, A. R.; Garcia-Suarez, V. M.; Bailey, S. W.; Lambert, C. J.; Ferrer, J.; Sanvito, S. Towards molecular spintronics. Nat. Mater. 2005, 4, 335-339.

(37) Rocha, A. R.; García-Suárez, V.; Bailey, S.; Lambert, C.; Ferrer, J.; Sanvito, S. Spin and molecular electronics in atomically generated orbital landscapes. Phys. Rev. B 2006, 73.

(38) Senn, H. M.; Thiel, W. QM/MM Methods for Biomolecular Systems. Angew. Chem., Int. Ed. 2009, 48, 1198-1229.

(39) Brunk, E.; Rothlisberger, U. Mixed Quantum Mechanical/Molecular Mechanical Molecular Dynamics Simulations of Biological Systems in Ground and Electronically Excited States. Chem. Rev. 2015, 115, 6217-6263, PMID: 25880693.

(40) Soler, J. M.; Artacho, E.; Gale, J. D.; García, A.; Junquera, J.; Ordejón, P.; Sánchez-Portal, D. The SIESTA Method for ab initio Order-N Materials Simulation. J. Phys. Cond. Mat. 2002, 14, 2745.

(41) Sanz-Navarro, C. F.; Grima, R.; García, A.; Bea, E. A.; Soba, A.; Cela, J. M.; Ordejón, P. An efficient implementation of a QMMM method in SIESTA. Theoretical Chemistry Accounts 2010, 128, 825-833.

(42) Crespo, A.; Scherlis, D. A.; Marti, M. A.; Ordejon, P.; Roitberg, A. E.; Estrin, D. A. "A DFT-based QM-MM approach designed for the treatment of large molecular systems: Application to chorismate mutase". J. Phys. Chem. B 2003, 107, 13728-13736.

(43) Merchant, C. a.; Healy, K.; Wanunu, M.; Ray, V.; Peterman, N.; Bartel, J.; Fischbein, M. D.; Venta, K.; Luo, Z.; Johnson, a. T. C. et al. DNA translocation through graphene nanopores. Nano Lett. 2010, 10, 2915-21. 
(44) Schneider, G. F.; Kowalczyk, S. W.; Calado, V. E.; Pandraud, G.; Zandbergen, H. W.; Vandersypen, L. M. K.; Dekker, C. DNA translocation through graphene nanopores. Nano Lett. 2010, 10, 3163-7.

(45) Hornak, V.; Abel, R.; Okur, A.; Strockbine, B.; Roitberg, A.; Simmerling, C. Comparison of multiple Amber force fields and development of improved protein backbone parameters. Proteins: Struct., Funct., Bioinf. 2006, 65, 712-725.

(46) Berendsen, H.; van der Spoel, D.; van Drunen, R. GROMACS: A messagepassing parallel molecular dynamics implementation. Comput. Phys. Commun. 1995, 91, 43-56.

(47) Darden, T.; York, D.; Pedersen, L. Particle mesh Ewald: An Nílog(N) method for Ewald sums in large systems. J. Chem. Phys. 1993, 98, 10089-10092.

(48) Berendsen, H.; Postma, J.; Gunsteren, W.; Hermans, J. In Intermolecular Forces; Pullman, B., Ed.; The Jerusalem Symposia on Quantum Chemistry and Biochemistry; Springer Netherlands: Netherlands, 1981; Vol. 14; pp 331-342.

(49) Hoover, W. G. Canonical dynamics: Equilibrium phase-space distributions. Phys. Rev. A 1985, 31, 1695-1697.

(50) Nose, S. A unified formulation of the constant temperature molecular dynamics methods. J. Chem. Phys. 1984, 81.

(51) Parrinello, M.; Rahman, A. Polymorphic transitions in single crystals: A new molecular dynamics method. J. Appl. Phys. 1981, 52.

(52) Perdew, J. P.; Burke, K.; Ernzerhof, M. Generalized Gradient Approximation Made Simple. Phys. Rev. Lett. 1996, 77, 3865-3868.
(53) McDaniel, J. G.; Schmidt, J. NextGeneration Force Fields from SymmetryAdapted Perturbation Theory. Ann. Rev. Phys. Chem. 2016, 67, 467-488, PMID: 27070322. 


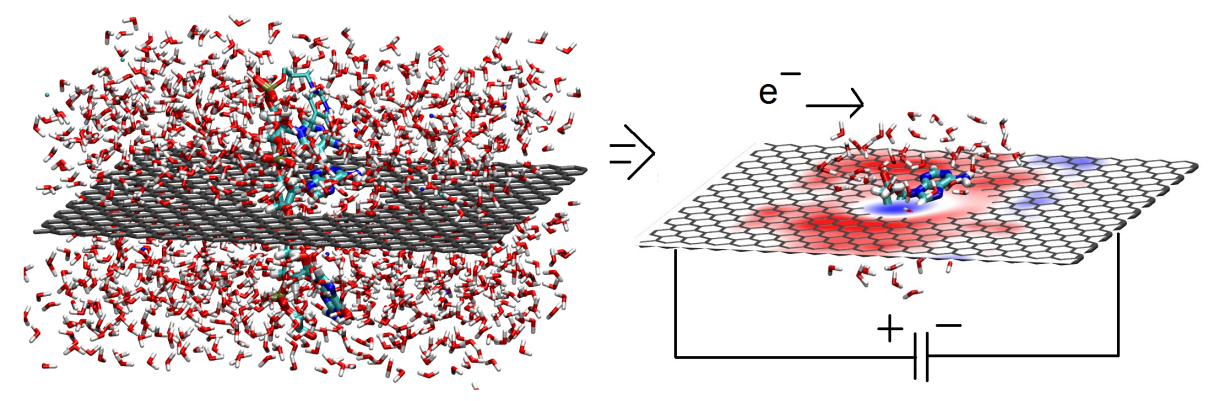

Figure 9: TOC Graphic 